\title{
Detection of Small for Gestational Age (SGA) newborn: a comparison of 'gold' standards
}

\author{
Liza Rose $^{1}$, Kathy Redfern ${ }^{2}$, Joanne Hosking $^{2}$, and Ross Welch ${ }^{3}$ \\ ${ }^{1}$ Derriford Hospital \\ ${ }^{2}$ Plymouth University \\ ${ }^{3}$ Affiliation not available
}

July 8, 2020

\begin{abstract}
Objective: Comparison of birthweight references for diagnosing SGA. To provide denominator data for suspicion and diagnosis of SGA. Design: A retrospective cohort study of 10,616 babies. Setting: A regional obstetric centre. Population: 10,616 consecutive newborns, born in Derriford Hospital, University Hospitals Plymouth NHS Trust (UPHT), whilst using the GROW package,1 compared with using Intergrowth 21st (IG21),2 and British 1990 (UK90) references.3 Methods: Statistical analysis of centile data from GROW, IG21 and UK90 references. Main outcomes: Induction rates, detection of suspected and/or diagnosed SGA. Assessment of goodness of fit to the Plymouth population. Results: GROW and IG21 showed bias. GROW had a systematic bias towards smaller centiles (skewness 0.169). IG21 had a systematic bias towards larger centiles (skewness 0.452). UK90 was best fit to the Plymouth dataset with insignificant bias across centiles (skewness -0.047). Conclusions: GROW and IG21 are not appropriate gold standards for our population for allocation of birthweight centile. The size of the population suggests the conclusions may be extrapolatable to other centres. UK90 does not have everyday accessible tools compared with GROW and IG21. A continual local audit of birthweight would be ideal, enabling accurate local centile allocation. If a national SGA screening programme monitoring units' ability to detect SGA was introduced, it could not start without validated, unit specific birthweight data. Funding: The statistician's funding was obtained from UHPT Research and Development generic funding.
\end{abstract}

\section{Abstract}

Objective : Comparison of birthweight references for diagnosing SGA. To provide denominator data for suspicion and diagnosis of SGA.

Design : A retrospective cohort study of 10,616 babies.

Setting : A regional obstetric centre.

Population : 10,616 consecutive newborns, born in Derriford Hospital, University Hospitals Plymouth NHS Trust (UPHT), whilst using the GROW package, ${ }^{1}$ compared with using Intergrowth $21^{\text {st }}$ (IG21), ${ }^{2}$ and British 1990 (UK90) references. ${ }^{3}$

Methods : Statistical analysis of centile data from GROW, IG21 and UK90 references.

Main outcomes : Induction rates, detection of suspected and/or diagnosed SGA. Assessment of goodness of fit to the Plymouth population.

Results : GROW and IG21 showed bias. GROW had a systematic bias towards smaller centiles (skewness 0.169). IG21 had a systematic bias towards larger centiles (skewness -0.452). UK90 was best fit to the Plymouth dataset with insignificant bias across centiles (skewness -0.047). 
Conclusions : GROW and IG21 are not appropriate gold standards for our population for allocation of birthweight centile. The size of the population suggests the conclusions may be extrapolatable to other centres. UK90 does not have everyday accessible tools compared with GROW and IG21. A continual local audit of birthweight would be ideal, enabling accurate local centile allocation. If a national SGA screening programme monitoring units' ability to detect SGA was introduced, it could not start without validated, unit specific birthweight data.

Keywords : Birthweight, centile, SGA, small for gestational age, LGA, large for gestational age, GROW, Intergrowth 21, IG21, British 1990 reference values, UK90

\section{Introduction :}

In 2013 the Royal College of Obstetricians and Gynaecologists (RCOG) released its revised guideline for The Investigation and Management of the SGA Fetus (SGA GTG). ${ }^{4}$ In 2016 NHS England released "Saving Babies' Lives" (SBL), a care bundle for reducing stillbirth. ${ }^{5}$ This required units to provide the SGA birth rate, detection rate, false positive and false negative rate.

The availability of packages like GROW, provided the required data. UHPT implemented SGA screening using GROW software in April 2015. The unit noticed a near doubling of the induction rate using the combination of GROW and the SGA GTG recommendations. It was perceived that GROW allocated more babies to the SGA group. The unit also noticed a massive increase in ultrasound examinations requested (after the 18-20 week anomaly scan), linked contemporaneously to use of GROW and actions required by the SGA screening programme. Confidence in the GROW package decreased. Monitoring of our Stillbirth rate had shown an almost halving of the rate prior to the introduction of GROW.

The inability to access patient data in the GROW programme locally, only summary data being available, prevented our local assessment of interventions. Alternatives to GROW were sought and a decision was made to exit GROW and move to IG21.

GROW has a significant cost compared with IG21, which including all the analysis tools, is open access, so there was a cost saving with the change.

The core principle of GROW providing 'customised' resources based on maternal demographics versus the uncustomised approach of IG21 were discussed. Neither the RCOG SGA guideline nor the SBL care package made a clear recommendation about customisation. The SGA GTG says, "No trials were identified that compared customised with non-customised symphysis fundal height (SFH) charts and thus evidence for their effectiveness on outcomes such as perinatal morbidity/mortality is lacking". It also says, "Use of a customised fetal weight reference may improve prediction of a SGA neonate (good practice point)". In SBL the only reference is a footnote which says "Customised or other established growth chart"

This lack of evidence combined with a potentially adverse impact from GROW allowed a change. IG21 was implemented at booking in $13^{\text {th }}$ November 2018 replacing GROW in our local SGA guideline and practice.

\section{Methods:}

At delivery the birthweight and gestation were recorded on the GROW software. The software also required the reporting midwife to tick a field to say if the pregnancy had been 'suspected' of SGA or growth restriction or if either had been 'detected'. A departmental standard policy was used to answer both questions (online Box A). Routine data collection included whether the delivery was induced or not.

We requested, in June 2018, from the GROW programme, all the individual patient data that had been entered during our use. After the last birthweights were entered, we received the whole dataset in June 2019. The dataset identified patients only by their GROW identity number (GROW ID), which was generated as the patient was entered on the programme at their 12 week scan and contained no patient identifiers. All analyses were done using the GROW ID as the only identifier. The GROW dataset provided the gestation (days), birthweight (grammes), the calculated customised GROW centile and the status at birth 
(live/stillbirth). It also provided the response to the delivery questions about 'suspicion' or 'detection' of SGA (online Box A). GROW does not produce information about high birthweights other than centile.

The IG21 Neonatal Size Calculator (newborn infants between $24^{+0}$ and $42^{+6}$ weeks gestation) app ${ }^{6}$ was used on the same dataset to derive the IG21 birthweight centile and Z score. The same approach was used to derive the UK90 birthweight centile and Z score using a free tool (LMSgrowth programme, v2.77 authors Huiqi Pan and Tim Cole, copyright MRC 2002-12). ${ }^{7}$ The UK90 references were reanalysed in $2009^{8}$ when new UK-WHO growth charts were introduced. Review by the joint committee of the Royal College of Paediatrics and Child Health and the Scientific Advisory Committee on Nutrition, concluded the UK90 reference should be retained for assessment at birth, as the WHO mean birth weight for term infants was considerably lower than for UK infants, and the WHO values omit preterm births. ${ }^{9}$

This provided us with a single large dataset, with each patient identified by GROW ID, but with centiles from GROW, IG21 and UK90 references. We derived Z scores from IG21 and UK90 we were not provided with $\mathrm{Z}$ scores for the GROW data calculations.

We examined the distribution of the centiles (by plotting histograms and calculating corresponding skewness values) and the number of births deemed SGA (birthweight $<10^{\text {th }}$ centile) according to each of the reference standards. Analysis was carried out in R. ${ }^{10}$

\section{Results:}

For all analyses we excluded the 40 stillborn infants (overall cohort singleton stillbirth rate $=3.77 / 1000$ ) as both the cause of the stillbirth and the time from diagnosis to delivery may affect the weight at delivery and thus the allocated centile. This resulted in a cohort of 10576 liveborn singletons.

We plotted frequency histograms of birthweight centiles for each reference standard. In the absence of bias, the centiles would be expected to be uniformly distributed (showing approximately equal numbers of observations in each bar of the histogram and a skewness value near zero).

The histogram of the GROW centiles (Figure 1) confirmed a systematic bias, with a positive (right) skew of 0.169 , towards allocating birthweights to a lower (customised) centile when used on our local population .

We found the opposite bias with the IG21 centile centiles (Figure 1), with a negative (left) skew of -0.452 .

The UK90 reference range gave the most uniform distribution of centiles (Figure 1) with a minor negative left skew of -0.047 , for our population.

The Saving Babies lives care bundle requires units to report to NHS England their SGA, true positive rate and false negative rates. These values were calculated for the population by each package (Table 1). The rate of SGA derived from each package varied from $4.9 \%$ to $11 \%$ with newborn SGA numbers varying from 518 for IG21 to 1167 for GROW. IG21 gave the lowest true positive rate (32.8\%) and highest false negative rate $(67.2 \%)$. The UK90 analysis produced the highest true positive rate identifying 427 (59.4\%) SGA births with the lowest false negative rate $(40.8 \%)$.

A previous unpublished internal review of 200 consecutive stillbirths at UPHT, over a decade, showed our singleton stillbirth rate to have fallen from 5.84/1000 in 2011 to 1.98/1000 in 2013 (figure 2). Ultrasound examination numbers (post routine anomaly scan) were stable at 15-16,000 between 2010 and 2014 but increased between 2015 and 2017 to over 21100 (figure 3). Labour induction rate was calculated as part of our continuing quality dashboard. It rose from $21 \%$ in Dec 2014 to a peak of $36.4 \%$ in July 2018 (online figure A).

The birthweight (online figure B) and gestation data (online figure C) produced the expected left skewed distributions. The median birthweight was 3425 grammes (interquartile range: $3100-3750 \mathrm{~g}$ ). Median gestation was 277 days (interquartile range: $271-284$ days). The prematurity rate ( $<37$ weeks) was $5.4 \%$.

\section{Discussion}


An increase in clinical intervention by early delivery, including by labour induction, associated with the SGA GTG guideline and the introduction of the GROW package was evidenced by the rapid rise in average monthly induction rate from $21 \%$ in January 2015 pre introduction, to $31.5 \%$ in January 2016 and a peak of $36.4 \%$ in July 2018. An overall increase of $73 \%$ more inductions contemporaneous with the introduction of GROW. There may have been other components to this increase but there were no other changes in clinical policies in the unit.

The purpose of SBL was to reduce stillbirths. The Plymouth singleton stillbirth rate had fallen between 201013, believed to be due to the introduction of a RCOG Certificate of Completed Training holder (Consultant or Fellow), resident on delivery suite, including every night, for 164 hours a week in 2012. The Stillbirth rate did not show a significant change following the introduction of GROW and the SGA GTG. Introduction of GROW was also contemporaneously associated with a massive rise in the number of (post anomaly) growth ultrasound scans. This put enormous pressure on the obstetric ultrasound service and Fetal Medicine unit who had to provide $40 \%$ scans.

The SGA GTG changed the primary aim of fetal wellbeing screening from detection of fetal growth restriction (FGR) to detection of SGA fetuses, despite the guideline identifying that the majority of SGA fetuses will be normal (constitutionally) small. Concentration on detecting SGA fetuses would not identify FGR fetuses who were destined to be in the upper 50 percentiles but were growth restricted to a lower centile, unless they went below the tenth centile. The majority of FGR fetuses will not be detected by simply detecting SGA fetuses.

Since moving to IG21, the induction rate rise stopped, and the numbers of infants allocated as SGA decreased. The question is whether neither GROW or IG21 are "correct". The UK90 reference ranges were adopted across the UK in the late 1990s ${ }^{8,10}$ and were reanalysed in $2009^{3}$ for inclusion in the UK-WHO growth charts, still the standards in the Personal Child Health Record (red book). There are more recent gender specific UK birthweight centiles ${ }^{11}$ but these have not been adopted nationally. There is no software package analogous to GROW or IG21, useable on a daily basis to allocate SGA at birth for either UK90 or others. Of the three birthweight references tested, the UK90 gave the best true positive and least false negative rates. It would appear to be the most appropriate for our routine use.

Screening for fetal wellbeing is a complex process. The primary screening tools are patient history and SFH measurement. SFH has been in use for 50 years ${ }^{11}$ and there are over 20 uncustomised SFH references in use ${ }^{12}$ but only one customised. ${ }^{13}$ If ultrasound is indicated there are multiple charts for each part of the fetus. In the version of ultrasound database (Viewpoint 5.6.25.281, GE Healthcare, UK) we are currently using there are 17 formulae available for calculating estimated fetal weight (EFW). UPHT policy dictates the Hadlock four parameter (biparietal diameter, head circumference, abdominal circumference and femur length) formula ${ }^{14}$ which has been published as the most accurate, although accuracy of EFW is at best \pm $10-20 \%$, with a systematic over estimation. ${ }^{15}$

The GROW process compiles the errors at each step in the process. If a fetus is suspected of being small by $\mathrm{SFH}$, with it's inter and intraobserver error, an ultrasound is performed. The abdominal circumference and EFW are calculated, each with their own inter and intraobserver error dependant on the formulae used. The EFW is then manually transferred to the GROW SFH/EFW paper chart stored in the patient's notes, with manual transcription errors combined with plotting errors due to the size of the chart. The GROW EFW has its own error range. At delivery the newborn is weighed and the weight is entered onto GROW which has another reference range with its errors.

When the change was made to the IG21 process, we introduced IG21 SFH charts, changed our ultrasound database to use IG21 fetal biometry references and entered the birthweight into the IG21 software, thus using references all derived from one dataset ${ }^{16}$ in the hope of reducing accumulated errors. The finding that the IG21 process introduced a significant a skew to the allocation, albeit in the opposite direction to GROW, was disappointing.

The focus of all the additional work introduced by SBL and SGA GTG was to reduce avoidable stillbirth. An 
aim all clinicians support fully. The review of 200 consecutive stillbirths, identified that there were similar numbers of stillbirths who were suspected of being large for gestational age as there were who were SGA. In addition several stillbirths were 'expected', either as a result of parental choice to interrupt (e.g. delayed selective feticide giving the survivor twin a better chance at later gestation; feticide at $<24$ weeks but delivery occurring after 24 weeks) or where parental choice was to allow a fetus to die rather than actively end the pregnancy. With 10-12 stillbirths per year a small number of expected cases has a significant effect on the total rate, so the process set in place over the past decade by external guidance, concentrating solely on detection of SGA rather than fetal health will always have limitations in reducing avoidable stillbirths.

Screening for SGA is not a Public Health England Screening programme, unlike screening for trisomies or the anomaly scan. ${ }^{17}$

A future larger multicentre study should link neonatal outcome with antenatal policies to determine which predict poor outcomes, rather than simply being sizeist.

This study suggests that maternity units should assess the impact on local intervention rates, clinical outcomes and changes in workload associated with introduction of programmes like GROW and Intergrowth 21, but also hints at the need for a centrally organised, properly funded, PHE specified programme of assessment of fetal growth screening. A central part of such a programme would be validated birthweight centiles. As in the trisomy programme ${ }^{17}$, sonographers should be centrally monitored and recertified for biometry measurements. Standardised ultrasound measurements and EFW formulae using appropriate longitudinal statistical analysis ${ }^{18,19}$ should be used in all units. This approach is already standard for both Crown Rump Length and Nuchal Translucency measurements and there is a national quality assurance programme monitoring the process, with local and national reporting. Surely, if the aim is truly to reduce stillbirth and perhaps further, to improve all fetal heath monitoring, such a national Growth screening programme is now required.

\section{Conclusions}

This cohort study demonstrated that introduction of software to obtain reportable data may lead to significant changes in intervention potentially without patient benefit. It suggests that all units should review the software they are using and assess whether the references fit their population or introduce significant bias that alters clinical behaviour and potentially increases clinical harm. The study recommends that for the Plymouth population the UK90 birthweight reference would be the best fit, but it does not currently have the user friendly tools needed for everyday clinical use

\section{Required Statements}

\section{Disclosure of Interest:}

The authors declare there are no conflicts of interest

\section{Contribution of authors:}

CRW conceived the idea, collated data and was the primary writer of the paper. L Rose coordinated data gathering, clinical practice and contributed to the paper writing. KMR coordinated the UK-WHO data analysis and contributed to the paper writing. JH performed all statistical analysis and contributed to the paper writing.

\section{Details of Ethics approval:}

As this study is a secondary use of anonymised data (see line 84) collected by staff within a care team in the course of care for their own patients or clients (without an intention to use it for research at the time of collection) ethics committee approval was not required.

Funding: The statistician's funding was obtained from UHPT Research and Development generic funding. No other funding was required.

\section{Reference List:}


1. Gardosi J. GROW Documentation [Internet]. 2019. Available from: https://www.gestation.net/GROW_documentation.pdf

2. INTERGROWTH-21st. The International Fetal and Newborn Growth Consortium for the 21st Century [Internet]. 2020. Available from: https://intergrowth21.tghn.org/

3. Cole TJ, Williams AF, Wright CM. Revised birth centiles for weight, length and head circumference in the UK-WHO growth charts. Ann Hum Biol;38(1):7-11.

4. Royal College of Obstetricians and Gynaecologists. Small-for-Gestational-Age Fetus, Investigation and Management (Green-top Guideline No. 31) [Internet]. 2013. Available from: https://www.rcog.org.uk/en/guidelines-research-services/guidelines/gtg31/

5. NHS England. Saving Babies' Lives A care bundle for reducing stillbirth [Internet]. 2016. Available from: https://www.england.nhs.uk/wp-content/uploads/2016/03/saving-babies-lives-car-bundl.pdf

6. INTERGROWTH-21st. Neonatal Size Calculator for newborn infants between $24+0$ and $42+6$ weeks' gestation [Internet]. 2020. Available from: https://intergrowth21.tghn.org/newborn-size-birth/\#c4

7. Cole TJ, Green PJ. Smoothing reference centile curves: The lms method and penalized likelihood. Stat Med. 1992. 11(10):1305-19.

8. Cole TJ, Freeman J V., Preece MA. British 1990 growth reference centiles for weight, height, body mass index and head circumference fitted by maximum penalized likelihood. Stat Med. 1998;17(4):407-29.

9. SACN/RCPCH Expert Group. Application of WHO Growth Standards in the UK [Internet]. London: The Stationery Office; 2007. Available from: https://www.gov.uk/government/uploads/system/uploads/attachment_data/file/338922/SACN_RCPCH_Application_of_WHO_Growth_Standards.pdf

10. R Core Team (2019). R: A language and environment for statistical computing. R Foundation for Statistical Computing, Vienna, Austria. URL https://www.R-project.org/.

11. Norris T, Seaton SE, Manktelow BN, et al Updated birth weight centiles for England and Wales Archives of Disease in Childhood - Fetal and Neonatal Edition 2018;103:F577-F582.

11. Calvert JP, Crean EE, Newcombe RG, Pearson JF. Antenatal screening by measurement of symphysisfundus height. BMJ [Internet]. 1982; 285(6345):846-9.

12. Papageorghiou AT, Ohuma EO, Gravett MG, Hirst J, da Silveira MF, Lambert A et al. International standards for symphysis-fundal height based on serial measurements from the Fetal Growth Longitudinal Study of the INTERGROWTH-21st Project: prospective cohort study in eight countries BMJ 2016; 355 :i5662

13. Mongelli M, Gardosi J. Symphysis-fundus height and pregnancy characteristics in ultrasound-dated pregnancies. Obstet Gynecol. 1999 Oct 1;94(4):591-4.

14. Hadlock FP, Harrist RB, Sharman RS, Deter RL, Park SK. Estimation of fetal weight with the use of head, body, and femur measurements-A prospective study. Am J Obstet Gynecol. 1985 Feb 1;151(3):333-7.

15. Robson SC, Gallivan S, Walkinshaw SA, Vaughan J, Rodeck CH. Ultrasonic estimation of fetal weight: use of targeted formulas in small for gestational age fetuses. Obstet Gynecol. 1993 Sep;82(3):359-64.

16. Villar J, Papageorghiou AT, Pang R, Ohuma EO, Ismail LC, Barros FC, et al. The likeness of fetal growth and newborn size across non-isolated populations in the INTERGROWTH-21st project: The fetal growth longitudinal study and newborn cross-sectional study. Lancet Diabetes Endocrinol. 2014;2(10):781-92.

17. Public Health England. NHS fetal anomaly screening programme (FASP) [Internet]. 2013. Available from: https://www.gov.uk/topic/population-screening-programmes/fetal-anomaly 
18 Royston P. Constructing time-specific reference ranges. Stat Med. 1991; 10(5):675-90. doi:10.1002/sim.4780100502

19 Royston P. Calculation of unconditional and conditional reference intervals for foetal size and growth from longitudinal measurements. Stat Med. 1995;14(13):1417-36. doi:10.1002/sim.4780141303

Table A

Rate of SGA, True Positive rate and false Negative rate for each reference used

\begin{tabular}{lllll} 
& SGA detected $(\mathbf{n})$ & SGA rate \% & TP rate \% & FN rate \% \\
\hline GROW & 1167 & 11.0 & 53.7 & 46.3 \\
IG21 & 518 & 4.9 & 32.8 & 67.2 \\
WHO & 968 & 9.2 & 59.4 & 40.6 \\
\hline
\end{tabular}

\section{Hosted file}

Figure 1.docx available at https://authorea.com/users/340996/articles/467987-detection-ofsmall-for-gestational-age-sga-newborn-a-comparison-of-gold-standards

\section{Hosted file}

Figure 2.docx available at https://authorea.com/users/340996/articles/467987-detection-ofsmall-for-gestational-age-sga-newborn-a-comparison-of-gold-standards

\section{Hosted file}

Figure 3.docx available at https://authorea.com/users/340996/articles/467987-detection-ofsmall-for-gestational-age-sga-newborn-a-comparison-of-gold-standards 